\title{
Convergence of Quintic Spline Interpolation
}

\author{
Y.P. Dubey \\ Department Of Mathematics, \\ L.N.C.T. \\ Jabalpur 482003
}

\author{
Anil Shukla \\ Department Of Mathematics \\ Gyan Ganga College Of Technogy, \\ Jabalpur 482003
}

\begin{abstract}
In this paper, we have investigate existance, uniqueness and error bound of quintic spline interpolation.
\end{abstract}

Key words : $\quad$ Convergence, Quintic spline, Interpolation, Error Bound, Deficient.

\section{INTRODUCTION}

Piecewise Quartic, Quintic and higher degree spline are popular for smooth and best approximation see Deboor [1]. Interpolation by lower degree spline are widely used in the method of Piecewise Polynomial approximation to represent a non analytic funciton. Interpolation by Quintic and higher degree spline, the maximum error between a function and its interpolant can be controlled by mesh spacing and such function have no corner at the joint of two pieces and therefore no more data are required than lower order method to get desire accuracy. Therefore, quintic and higher degree spline are useful than lower degree spline for best approximation See [1]. In the direction of more higher degree spline Jianzhone and Hung [9] have obtained optimal error bounds for quartic and quintic interpolatory splines (see also Howell and Verma) [2] Wong and Agrawal [5], Gmelling Meling [6] have obtained explicit error bounds for quintic and biquintic interpolatory spline Dubey [10] has obtained existence, uniqueness and error bound for higher degree spline (See also Rana and Dubey) [8]. In the present paper we shall obtain precise error estimate of deficient quintic spline interpolation matching the given function values at mesh points and its derivative at two interior points of the interval.

\section{EXISTENCE AND UNIQUENESS}

Consider a mesh $\mathrm{P}$ of $[0,1]$ given by $0=\mathrm{x}_{\mathrm{o}}<\mathrm{x}_{1}, \ldots,<\mathrm{x}_{\mathrm{n}}=1$ such that $x_{i+1}-x_{i}=h_{i}, i=0,1, \ldots, n-1$. For a positive integer $\mathrm{m}$, let $\pi_{5}[0,1]$ denote the set of all real algebraic polynomials of degree less than or equal to $\mathrm{m}_{5}$. For a function $\mathrm{s}$ defined over $\mathrm{P}$. we denote the restriction of $\mathrm{s}$ over $\left[\mathrm{x}_{\mathrm{i}}, \mathrm{x}_{\mathrm{i}+1}\right]$ by $\mathrm{s}$. The class $\mathrm{R}(5,2, \mathrm{P})$ of deficient quintic splines defined over $\mathrm{P}$ is given by

$$
\mathrm{R}(5,2, P)=\left\{s: s \varepsilon C^{2}[0,1], s_{\mathrm{i}} \varepsilon \pi_{5}, i=0,1,2 n-1\right\},
$$

wherein $\mathrm{R} *(5,2, \mathrm{P})$ denotes the class of all deficient quintic splines $\mathrm{R}(5,2, \mathrm{P})$ which satisfy the boundary conditions

$$
s^{\prime}\left(x_{0}\right)=f^{\prime}\left(x_{0}\right), s^{\prime}\left(x_{n}\right)=f^{\prime}\left(x_{n}\right) .
$$

We introduce the following interpolatory conditions:

$$
\begin{aligned}
& s\left(x_{i}\right)=f\left(x_{i}\right), i=0,1, \ldots, n \\
& s^{\prime}\left(\alpha_{i}\right)=f^{\prime}\left(\alpha_{i}\right), i=1,2, \ldots ., n \\
& s^{\prime}\left(\beta_{i}\right)=f^{\prime}\left(\beta_{i}\right), \quad i=1,2, \ldots ., n
\end{aligned}
$$

where

$$
\begin{aligned}
& \alpha_{i}=x_{i-1}+(1 / 3) h_{i-1} \\
& \beta_{i}=x_{i-1}+(2 / 3) h_{i-1}
\end{aligned}
$$

In fact, we shall prove the following:

THEOREM 2.1. Let $f$ exist, then there exists a unique deficient quintic spline $s$ in $R^{*}(5,2, P)$ which satisfies the interpolatory conditions (2.1) - (2.3).

Proof : Considering a quintic polynomial $P(z)$ on $[0,1]$, it can be easily verified that

$E(z)=E(0) Q_{1}(z)+E^{\prime}(1) \cdot Q_{2}(z)+E^{\prime}(1 / 3) \cdot Q_{3}(z)+E^{\prime}(2 / 3)$. $Q_{4}(z)+E^{\prime}(O) Q_{5}(z)+E^{\prime}(1) \cdot Q_{6}(z)$

where

$$
\begin{aligned}
& Q_{1}(z)=\frac{1}{4}\left[4-120 z^{2}+440 z^{3}-540 z^{4}+216 z^{5}\right] \\
& Q_{2}(z)=\frac{1}{4}\left[120 z^{2}-440 z^{3}+540 z^{4}-216 z^{5}\right] \\
& Q_{3}(z)=\frac{1}{4}\left[-27 z^{2}+135 z^{3}-189 z^{4}+81 z^{5}\right] \\
& Q_{4}(z)=\frac{1}{4}\left[-54 z^{2}+189 z^{3}-216 z^{4}+81 z^{5}\right] \\
& Q_{5}(z)=\frac{1}{4}\left[4 z-8 z^{2}-23 z^{3}+54 z^{4}-27 z^{5}\right] \\
& Q_{6}(z)=\frac{1}{4}\left[-15 z^{2}+51 z^{3}-63 z^{5}+27 z^{5}\right]
\end{aligned}
$$

Now writing $t=\left(x-x_{i}\right) / h_{i}, o \leq t \leq 1$, (2.4) may be expressed in terms of the restriction $\mathrm{s}_{\mathrm{i}}$ of $\mathrm{s}$ on $\left[\mathrm{x}_{i}, \mathrm{x}_{i+1}\right]$ as follows :

$$
\begin{aligned}
& s_{i}(x)=f\left(x_{i}\right) \cdot Q_{1}(t)+f^{\prime}\left(x_{i+1}\right) \cdot Q_{2}(t)+f^{\prime}\left(\alpha_{i+1}\right) \cdot Q_{3}(t)+f\left(\beta_{i+1}\right) . \\
& Q_{4}(t)+h_{i} s^{\prime}\left(x_{i}\right) \cdot Q_{5}(t)+h_{i} s^{\prime}\left(x_{i+1}\right) \cdot Q_{6}(t)
\end{aligned}
$$

which clearly satisfies the conditions (2.1) - (2.3) and $s_{i}(x)$ is quintic in $\left[x_{i}, x_{i+1}\right]$ for $\mathrm{i}=0, \ldots ., \mathrm{n}-1$. Now observing the fact that $\mathrm{s} \in C^{2}[0,1]$, therefore, applying continuity condition of second derivative of $\mathrm{s}$ in (2.5), we get the following system of equations : 


$$
\begin{aligned}
& -\frac{23}{2} h_{i} s_{i-1}^{\prime}+\left(15 h_{i}+4 h_{i-1}\right) s_{i}^{\prime}+\frac{15}{2} s_{i+1}^{\prime} \\
& =F_{i}, i^{\prime}=1,2, \ldots . ., n-1
\end{aligned}
$$

where

$$
\begin{aligned}
F_{i}=60 & f_{i}\left(\frac{h_{i}}{h_{i-1}}-\frac{h_{i-1}}{h_{i}}\right)+60\left(\frac{h_{i-1}}{h_{i}} f_{i+1}-\frac{h_{i}}{h_{i-1}} f_{i-1}\right) \\
& -27\left(f^{\prime}\left(\alpha_{i+1}\right)+f^{\prime}\left(\beta_{i+1}\right)\right) h_{i-1} \\
& -27 h_{i}\left(f^{\prime}\left(\alpha_{i}\right)+f^{\prime}\left(\beta_{i}\right)\right)
\end{aligned}
$$

Since $\quad \frac{7}{2}\left(h_{i}-h_{i-1}\right)>0$ for $h_{i} \geq h_{i-1}$

It may be seen easily that the coefficient matrix of the system of equations (2.6) is diagonally dominant and hence invertible. This completes the proof of Theorem 2.1.

\section{ERROR BOUNDS}

In this section of the paper, we shall estimate the bounds of error function by using method of Hall and Meyer [2] and of its derivatives, i.e. $e^{(r)}(x)=s^{(r)}(x)-f^{(r)}(x), r=0,1$ for the spline interpolant of Theorem 2.1 which are best possible. Let $s(x)$ be the twice continuously differentiable quintic spline function satisfying the conditions of Theorem 2.1. Now considering $f \in C^{(6)}[0,1]$ and denoting the unique quintic polynomial by $L_{i}[f, x]$ which agrees with given functional values and derivatives $f^{\prime}\left(\alpha_{i+1}\right), f^{\prime}\left(\beta_{i+1}\right), f^{(r)}\left(x_{i}\right)$ and $f^{(r)}\left(x_{i+1}\right)$, for $r=0,1$, we have for $x \in\left[x_{i}, x_{i+1}\right]$,

$|s(x)-f(x)| \cong\left|s_{i}(x)-f(x)\right| \leq\left|s_{i}(x)-L_{i}[f, x]\right|+\left|L_{i}[f, x]-f(x)\right|$

We now proceed to get pointwise bounds of both the terms on the right hand side of (3.1). The estimate of the second term can be obtained by following a well known remainder theorem for polynomial interpolation of Davis [4].

$\left|\mathrm{L}_{\mathrm{i}}[\mathrm{f}, \mathrm{x}]-\mathrm{f}(\mathrm{x})\right| \leq \frac{h_{i}^{6}}{6 !}\left|\mathrm{t}^{2}(1-\mathrm{t})^{2}(1-3 \mathrm{t})(2-3 \mathrm{t})\right| \mathrm{F} / 9$

Where

$$
t=\left(x-x_{i}\right) / h_{i}
$$

$$
\text { and } \quad F=\max _{0 \leq x \leq 1}\left|f^{6}(x)\right|
$$

We next turn over attention to get a similar bounds for $\mid s_{i}(x)-L_{i}$ $[f, x] \mid$. From (2.4), we have

$$
s_{i}(x)-L_{i}[f, x]=h_{i} e^{\prime}\left(x_{i}\right) \cdot Q_{5}(t)+h_{i} e^{\prime}\left(x_{i+1}\right) \cdot Q_{6}(t)
$$

Thus,

$$
\begin{aligned}
& \left|s_{i}(x)-L_{i}[f, x]\right| \leq h_{i}\left|e^{\prime}\left(x_{i}\right) \| Q_{5}(t)\right| \\
+ & h_{i} e^{\prime}\left(x_{i+1)}\right) \| Q_{6}(t) \mid .
\end{aligned}
$$

As

$$
\left|Q_{5}(t)\right|+\left|Q_{6}(t)\right|=\frac{1}{4}|t(1-t)| 19 t^{2}-19+4 \mid
$$

Thus, applying (3.5) in (3.4), we have

$\left|s_{i}(x)-L_{i}[f, x]\right| \leq h_{i} \max \left\{\left|e^{\prime}\left(x_{i}\right)\right|,\left|e^{\prime}\left(x_{i+1}\right)\right|\right\} k(t)$

where $\mathrm{k}(\mathrm{t})=\frac{1}{4}|t(1-t)| 19 t^{2}-19+4 \mid$

Let the max $\left|e^{\prime}\left(x_{i}\right)\right|$ exist for $i=j$, therefore (3.6) may be written as

$$
\left|s_{i}(x)-L_{i}[f, x]\right| \leq h\left|e^{\prime}\left(x_{i}\right) k(t)\right|
$$$$
0 \leq \mathrm{i} \leq \mathrm{n}
$$

Where $\mathrm{h}=\max \mathrm{h}_{\mathrm{i}}$, we now next proceed to find the upper bound for $\left|e^{\prime}\left(x_{i}\right)\right|$ needed in eq. (3.7). Replacing $s^{\prime}\left(x_{j}\right)$ by $e^{\prime}\left(x_{j}\right)$ in (2.6), we have

$$
\begin{gathered}
-\frac{23}{2} h_{j} e_{j-1}^{\prime}+\left(15 h_{j}+4 h_{j-1}\right) e_{j}^{i}+\frac{15}{2} h_{j-1} e_{j+1}^{\prime} \\
=F_{j}+\frac{23}{2} h_{j} f_{j-1}^{\prime}-\left(15 h_{j}+4 h_{j-1}\right) f_{j}^{\prime}-\frac{15}{2} h_{j-1} f_{j+1}^{\prime} \\
=\mathrm{B}(f)
\end{gathered}
$$

In view of the fact that $\mathrm{B}(f)$ is a linear functional which is zero for polynomial of degree 5 or less, we can apply the Peano Theorem Davis [4] to obtain

$$
B(f)=\int_{x_{j-1}}^{x_{j+1}} \frac{f^{(6)}(y)}{5 !} B\left[(x-y)_{+}^{5}\right] d y .
$$

Thus from (3.9), we get,

$|B(f)| \leq \frac{F}{5 !} \int_{f_{j-1}}^{x_{j+1}}\left|B\left[(x-y)_{+}^{5}\right]\right| d y$.

Further, it can be observed from (3.9) that for $x_{j-1} \leq y \leq x_{j+1}$

$$
\begin{aligned}
& B\left[(x-y)_{+}^{5}\right]=\left(15 h_{j}+4 h_{j-1}\right)\left(x_{j}-y\right)_{+}^{4} \\
& -\frac{75}{2} h_{j-1}\left(x_{j+1}-y\right)^{4}+60\left(x_{j}-y\right)_{+}^{5} \\
& \left(\frac{h_{j}}{h_{j-1}}-\frac{h_{j-1}}{h_{j}}\right)+60\left(\frac{h_{j-1}}{h_{j}}\left(x_{j+1}-y\right)^{5}\right) \\
& -135\left\{\left(\alpha_{j+1}-y\right)^{5}+\left(\beta_{j+1}-y\right)^{4}\right\} \\
& h_{j-1}-135\left\{\left(\alpha_{j}-y\right)^{4}+\left(\beta_{j}-y\right)^{4}\right\}
\end{aligned}
$$


In order to estimate the integral of r.h.s. of (3.10), we rewrite the above expression in the following symmetric form about $x_{j}$ to get.

$$
\begin{aligned}
& B\left[(x-y)_{+}^{5}\right]=\frac{h_{j}}{h_{j-1}}\left[-\frac{45}{2} h_{j-1}+60\left(x_{j}-y\right)\right] \\
& {\left[\left(x_{j}-y\right)-h_{j-1}\right]^{4} \quad x_{j-1} \leq y \leq \alpha_{j}} \\
& =\frac{h_{j}}{h_{j-1}}\left[\frac{255}{2} h_{j}\left(x_{j}-y\right)^{4}-90\left(x_{j}-y\right)^{3} h_{j-1}\right. \\
& \left.+15\left(x_{j}-y\right)^{2} h_{j-1}^{3}+10\left(x_{j}-y\right) h_{j-1}^{4}-25 h_{j-1}^{5}\right] \\
& \alpha_{j} \leq y \leq \beta_{j} \\
& \frac{h_{j}}{h_{j-1}}\left[\frac{-15}{2} h_{j-1}\left(x_{j}-y\right)^{4}+90 h_{j-1}^{2}\left(x_{j}-y\right)^{3}\right. \\
& -75\left(x_{j}-y\right)^{2} h_{j-1}^{3}+30 h_{j-1}^{2}\left(x_{j}-y\right)^{3} \\
& \left.\left(x_{j}-y\right) \frac{-80}{3} h_{j-1}^{3}\right] \\
& \beta_{j} \leq y \leq x_{j} \\
& =-\frac{h_{j-}}{h_{j}}\left[60\left(x_{j}-y_{1}\right)^{5}\right. \\
& +\frac{15}{2} h_{j}\left(x_{j}-y\right)^{4}-90 h_{j}^{2}\left(x_{j}-y\right)^{3} \\
& \left.+75\left(x_{j}-y\right)^{2} h_{j}^{3}+30 h_{j}^{4}\left(x_{j}-y\right)-\frac{80}{3} h_{j}^{5}\right] \\
& x_{j} \leq y \leq \alpha_{j+1} \\
& =\frac{h_{j-1}}{h_{j}}\left[60\left(x_{j}-y\right)^{5}+\frac{255}{2} h_{j}\left(x_{j}-y\right)^{4}\right. \\
& +90\left(x_{j}-y\right)^{3} h_{j}+15\left(x_{j}-y\right)^{2} h_{j}^{3} \\
& \left.-10\left(x_{j}-y\right) h_{j}^{4}-25 h_{j}^{5}\right] \\
& \alpha_{j+1} \leq y \leq \beta_{j+1} \\
& =\frac{h_{j-1}}{h_{j}}\left[\frac{45}{2} h_{j}+60\left(x_{j}-y\right)\right]\left[x_{j}-y+h_{j}\right]^{4} \\
& \beta_{j+1} \leq y \leq x_{j+1}
\end{aligned}
$$

From the above expression, it follows that

$$
\int_{x_{j-1}}^{x_{j+1}}\left|B\left[(x-y)_{+}^{5}\right]\right| d y=256.72 h_{j} h_{j-1}\left(h_{j}^{4}+h_{j-1}^{4}\right)
$$

Combining (3.10) with (3.11), we have

$$
|B(f)| \leq \frac{256.72 h_{j} \cdot h_{j-1}}{5 !}\left[h_{j}^{4}+h_{j-1}^{4}\right] F
$$

Thus from (3.8) and (3.12), it follows that

$$
\left|e^{\prime}\left(x_{j}\right)\right|=\left|e_{j}^{\prime}\right| \leq \frac{256.72 h_{j} \cdot h_{j-1}\left[h_{j}^{4}+h_{j-1}^{4}\right]}{5 !\left[\frac{7}{2} h_{j}+\frac{23}{2} h_{j-1}\right]}
$$

Now using (3.2), (3.7) along with (3.13) in (3.1), we have

$$
\begin{aligned}
& |e(x)| \leq \frac{h^{6}}{(5 !)} \mid t^{2}(1-t)^{2}(1-3 t)(2-3 t) F / 9 \\
& +h\left|e^{\prime}\left(t_{j}\right)\right| t(1-t)\left(9 t^{2}-19 t+4\right) \mid \\
& \leq \frac{h^{6}}{(5 !)} F|C(t)|
\end{aligned}
$$

Where

$$
\begin{aligned}
& \mid C(t)=F t(1-t)\left[(1-3 t)(2-3 t)+\left(9 t^{2}-19 f+4\right) / 15\right] \\
& =K^{*}(t)
\end{aligned}
$$

THEOREM 3.1. Let $s(x)$ be the quintic spline interpolant of Theorem 2.1 interpolating a given function $f(x)$ and $f \in C^{6}[0,1]$ then,

$$
|e(x)| \leq K_{0} h^{6} / 5 ! \max _{0 \leq x \leq 1}\left|f^{(6)}(x)\right|
$$

Where $K_{0}=\max _{0 \leq x \leq 1}|C(t)|$, defined by the equation (3.15)

Also, we have

$\left|e^{\prime}\left(x_{1}\right)\right| \leq K_{1} h^{5} / 5 ! \max _{0 \leq x \leq 1}\left|f^{(6)}(x)\right|$,

Where $\mathrm{K}_{1}=412 / 15$

Furthermore, it can be seen easily that $\mathrm{K}$ in (3.16) be improved for an equally spaced partition. Inequality (3.17) is also best possible. Also, we have

$\left|e^{\prime}(x)\right| \leq K_{1}\left(h^{5} / 5 !\right) \max _{0 \leq x \leq 1}\left|f^{(6)}(x)\right|$,

where $K_{2}$ is positive constant. Equation (3.14) proves (3.16) whereas inequality (3.17) is a direct consequence of (3.13). 
Now, we turn to see that the inequality (3.16) is best possible in the limit. Considering $f(x)=x^{6} / 6$ ! and using the Cauchy formula Davis [4], we have

$$
\begin{aligned}
& L\left[t^{6} / 6 !, x\right]-x^{6} / 6 !=h^{6}(1-t)^{2} t^{2} \\
& (t-1 / 3)(t-2 / 3) / 6 !
\end{aligned}
$$

Moreover, for the function under consideration (3.8) the following relation holds for equally spaced knots,

$$
B\left[\frac{x^{6}}{6 !}\right]=\frac{256.72}{5 !} h^{5}=-\frac{23}{2} e_{j-1}^{1}+19 e_{j}^{1}+\frac{15}{2} e_{j+1}^{1}
$$

Considering for a moment

$$
e^{\prime}\left(x_{i}\right)=\frac{256.72 h^{5}}{(5 !)(15)}=e^{\prime}\left(x_{i-1}\right)=e^{\prime}\left(x_{i+1}\right)
$$

we have from (3.4),

$$
\begin{aligned}
& s(x)-L_{i}[f, x]=\frac{256.72 h^{6}}{15(5 !)}\left(Q_{5}(t)+Q_{6}(t)\right) \\
& =\frac{256.72 h^{6}}{15(5 !)}\left[t(1-t)\left(9 t^{2}-19 t+4\right) / 4\right\}
\end{aligned}
$$

Now combining (3.19) and (3.22), we get for $x_{i} \leq x \leq x_{i+1}$

$$
\begin{aligned}
& s(x)-f(x)=\frac{h^{6}}{(5 !)}\left\{\frac{256.72}{15} t(1-t)\left\{\frac{9 t^{2}-19 t+4}{4}\right\}\right. \\
& \left.+\frac{1}{6} t(1-t)(t-1 / 3)(t-2 / 3)\right\}
\end{aligned}
$$

From (3.23), it is clearly observed that (3.16) is best possible, provided we could prove that

$$
e^{\prime}\left(x_{i-1}\right)=e^{\prime}\left(x_{i+1}\right)=e^{\prime}\left(x_{i}\right)=\frac{(256.72) h^{5}}{(5 !)(15)}
$$

In fact (3.24) is attained only in the limit. The difficulty will appear in the case of boundary conditions, i.e., $e^{\prime}\left(x_{0}\right)=e^{\prime}\left(x_{n}\right)=0$. However, it can be shown that as we move many subintervals away from the boundaries, $e^{\prime}\left(x_{i}\right) \rightarrow 256.72 h^{5} /(5 !)(15)$. For that, we shall apply (3.20) inductively to move away from the end conditions $e^{\prime}\left(x_{0}\right)=e^{\prime}\left(x_{n}\right)=0$.

The first step in this direction is to establish that $e^{\prime}\left(x_{i}\right) \geq 0$ for some $\mathrm{i}, \mathrm{i}=1, \ldots ., \mathrm{n}$ which can be shown by contradictory result, let $e^{\prime}\left(x_{i}\right)<0$ for some $\mathrm{i}=1,2 \ldots . \mathrm{n}-1$. Now making use of (3.17), we get

$$
\frac{256.72 h^{5}}{(5 !) 15} \geq-\frac{23}{2} e_{j-1}^{1}+19 e_{j}^{1}+\frac{15}{2} e_{j+1}^{1}
$$

for some $\mathrm{j}$ L.H.S. positive and r.h.s. is negative. Thus we have a contradication. Thus, $e^{\prime}\left(x_{i}\right) \geq 0$ for $\mathrm{i}=0, \ldots ., \mathrm{n}$.

Now from (3.20) we can write

$$
\begin{aligned}
& 19 e^{\prime}\left(x_{i}\right)=\frac{256.72 h^{5}}{(5 !)}-\left\{\frac{15}{2} e_{j+1}^{1}-\frac{23}{2} e_{j-1}^{1}\right\} \\
& =19 e^{\prime}\left(x_{j}\right) \leq \frac{256.72 h^{5}}{5 !}
\end{aligned}
$$

Since $e^{\prime}\left(x_{i}\right) \geq 0$, we get

$$
e^{\prime}\left(x_{i}\right) \leq \frac{256.72 h^{5}}{(5 !)(19)},
$$

Now again using (3.25) in (3.20), we have

$$
e^{\prime}\left(x_{i}\right) \leq \frac{256.72 h^{5}}{19(5 !)}\left\{1+\frac{4}{19}\right\} \quad \mathrm{i}=2,3, \ldots ., \mathrm{n}-2
$$

Thus, it is clear that a repeated use of (3.20) leads us to

$$
\left.e^{\prime}\left(x_{i}\right) \leq \frac{256.72 h^{5}}{19(5 !)}[1+4 / 19)+(4 / 19)^{2}+\ldots . .\right]
$$

Now, it can be seen easily that the right hand side of (3.26) $\rightarrow \frac{256.72 h^{5}}{15(6 !)}$ and hence in the limiting case.

$e^{\prime}\left(x_{i}\right) \leq \frac{256.72 h^{5}}{15(5 !)}$

which verifies the proof of (3.17).

Thus, corresponding to the function $f(x)=\frac{x^{6}}{(6 !)},(3.26)$ and (3.27) imply $e^{\prime}\left(x_{i}\right) \rightarrow \frac{256.72 h^{5}}{15(5 !)}$ in the limit for equally spaced knots. This completes the proof of Theorem 3.1

\section{REFERENCES}

[1] Deboor, C., A Practical Guide to splines, Applied Mathematical Science, Vol. 27 Springer - Verlag, New York, 1979.

[2] Hall, C.A. and Meyer, W.W., J. Approximation Theory, 16(1976), pp. 105-122.

[3] Howel, G. and Verma, A.K., Best Error Bond of Quartic Spline Interpolation, J. Appron Theory 58 (1989), 58-67. 
[4] Davis, P.J., Interpolation and approximation. New Yrok, 1961.

[5] Agrawal, R.P. and Wong, P.J.Y. Error Inqualities in Polynomial Interpolation and their application, Kumar Academic Publisher's (1993).

[6] Gemlling, R.H.J. and Meyling, G. In Interpolation by Bivariate Quintic splines of class .....(Construction in theory of functions 87), (Ed) Sendov et.al.) (1987), 15261.
[7] Rana, S.S., Dubey, Y.P. and Gupta, P. Convergence of Deficient Quintic Spline Interpolation, Journal of Indian Math Soc. Vol. No. 70 No.-1-4 (2003), pp. 41-28.

[8] Rana, S.S. and Dubey, Y.P. Best Error Bound of Quintic Spline Interpolation, J. Pure and app. Math 28 (10) $1937-$ 44, 1997.

[9] Jianzhong, W. and Hung, D. Scientia Sinica 24 (1982), 1130-41.

[10] Dubey, Y.P. Best Error Bound of Spline of Degree six, Int. Journal of Mathematics Analysis, Vol. 5, pp. 21-24, 2011. 